fluoroquinolones, had a strong activity to ciprofloxacin-resistant $\mathrm{N}$. gonorrhoeae strains. The MIC90 of ciprofloxacin or sitafloxacin were $16 \mu \mathrm{g} / \mathrm{ml}$ or $0.25 \mu \mathrm{g} / \mathrm{ml}$, respectively.

Purpose In this study, the relationship between genetic mutations of QRDR and antimicrobial susceptibilities of sitafloxacin against ciprofloxacin-resistant $\mathrm{N}$. gonorrhoeae strains was examined.

Methods The subjects were $12 \mathrm{~N}$. gonorrhoeae strains which were gotten by the Japanese national surveillance by three Japanese societies including the Japanese Association of Infectious Diseases, the Japanese Society of Chemotherapy and the Japanese Society of Clinical Microbiology. MICs of sitafloxacin to these 12 strains were more than $2 \mu \mathrm{g} / \mathrm{ml}$, but MICs of sitafloxacin to these strains were less than $0.125 \mu \mathrm{g} / \mathrm{ml}$. The base sequence of $\mathrm{ORDR}$ on gyrA or parC genes of these strains were examined

Results On ORDRof gyrA of 12 strains, mutations of 2 aminoacids were found, such as Ser91 to Phe, Asp95 to Ala or Asp95 to Gly. Regarding parC gene, mutations of 4 amino-acids were found, such as Asp86 to Asn in 1 strain, Ser87 to Asn in 6 strains, Ser87 to Arg in 5 strains, Glu91 to Lys, Gln or Gly in 3 strains and Ala123 to Ser in 3 strains.

Conclusion Sitafloxacin had a strong activity to ciprofloxacinresistant $\mathrm{N}$. gonorrhoeae which had at least more than 3 mutations of amino-acids on $\mathrm{QRDR}$ on gyrA and parC genes.

\section{P1.018 ANALYSIS OF MOSAIC PENICILLIN-BINDING PROTEIN 2 VARIANTS WITH ALA501 MUTATIONS THAT CONFER HIGH-LEVEL RESISTANCE TO EXPANDED-SPECTRUM CEPHALOSPORINS IN NEISSERIA GONORRHOEAE}

doi:10.1136/sextrans-2013-051184.0239

$1 \mathrm{~J}$ Tomberg, ${ }^{2} \mathbf{M}$ Unemo, ${ }^{3} \mathrm{C}$ Davies, ${ }^{1} \mathrm{R}$ Nicholas. ${ }^{1}$ University of North Carolina at Chapel Hill, Chapel Hill, NC, United States; ${ }^{2}$ Orebro University Hospital, Orebro, Sweden; ${ }^{3}$ Medical University of South Carolina, Charleston, SC, United States

Decreased susceptibility to the expanded-spectrum cephalosporins cefixime and ceftriaxone in Neisseria gonorrhoeae has increased dramatically over the past decade globally and recently resistance was reported to the last remaining recommended treatment option, ceftriaxone, raising fears that gonorrhoea may become untreatable. The major reason for decreased susceptibility has been mosaic penA alleles encoding penicillin-binding protein 2 ( $\mathrm{PBP} 2$ ), the major target for these antibiotics, with up to 70 mutations relative to wildtype. Less prevalent in most settings has been non-mosaic penA alleles containing A501V or A501T mutations just downstream of the KTG active site motif that also confer decreased susceptibility. We have shown that Ala501 mutations, when introduced into mosaic penA alleles, confer resistance to expanded-spectrum cephalosporins, and recently, a novel mosaic penA allele containing an A501P mutation and resulting in ceftriaxone resistance was described. To understand the role of Ala501 mutations in mosaic pen $A$ alleles, we transformed FA19 with the mosaic penA allele from $35 / 02$ harbouring a randomised codon at position 501 and selected for increased cefixime resistance. From this screen, we identified five Ala501 mutations (Val, Thr, Ser, Pro and Arg) that resulted in increased cefixime resistance, indicating that only a small subset of mutations are capable of conferring resistance. Surprisingly, only one clone with an A501P mutation was selected, perhaps suggesting a fitness defect with this mutation. MIC analyses showed that mutation of Ala501 to Val, Ser or Thr conferred 2.5-fold increases in resistance, whereas mutation to Arg and Pro increased resistance nearly 5-fold. PBP2-6140CT (PBP2 containing four C-terminal mutations) harbouring A501V or A501T mutations was crystallised and revealed major ordering and some reorganisation of the $\beta 3-\beta 4$ hairpin that is immediately adjacent to the active site. Modeling of $\beta$-lactams into the crystal structure indicates that the mutations likely introduce a steric clash with the R1 substituent of expanded-spectrum cephalosporins.

\section{P1.019 IDENTIFICATION OF THE AMINO ACIDS CONFERRING HIGH-LEVEL RESISTANCE TO EXPANDED-SPECTRUM CEPHALOSPORINS IN THE PENA GENE FROM THE NEISSERIA GONORRHOEAE STRAIN H041}

doi:10.1136/sextrans-2013-051184.0240

1J Tomberg, ${ }^{2} \mathbf{M}$ Unemo, ${ }^{3} \mathrm{M}$ Ohnishi, ${ }^{4} \mathrm{C}$ Davies, 'R Nicholas. 'University of North Carolina at Chapel Hill, Chapel Hill, NC, United States; ${ }^{2}$ Orebro University Hospital, Orebro, Sweden; ${ }^{3}$ National Institute of Infectious Diseases, Tokyo, Japan; ${ }^{4}$ Medical University of South Carolina, Charleston, SC, United States

The recent identification of a high-level ceftriaxone-resistant (MIC $=2-4 \mu \mathrm{g} / \mathrm{ml}$ ) isolate of Neisseria gonorrhoeae from Japan (H041) portends the loss of ceftriaxone as an effective treatment for gonococcal infections. This is of grave concern because ceftriaxone is the last remaining option for first-line empiric antimicrobial monotherapy. The penA gene from H041 (penA41) is a mosaic penA allele similar to mosaic penA alleles conferring intermediate-level cephalosporin resistance $\left(\mathrm{Ceph}^{\mathrm{I}}\right)$ worldwide, but has 13 additional mutations compared to the mosaic penA gene from the previously studied Ceph ${ }^{1}$ strain, 35/02 (penA35). When transformed into the wild-type strain FA19, the penA41 allele confers 300- and 570-fold increases in the MIC of ceftriaxone and cefixime, respectively. In order to understand the mechanisms involved in high-level ceftriaxone resistance and to improve the surveillance and epidemiology during the potential emergence of ceftriaxone resistance, we sought to identify the minimum number of amino acid alterations above those in penA35 that confer high-level resistance to ceftriaxone. Using restriction-fragment exchange and site-directed mutagenesis, we identified three mutations - A311V, T316P, and T483S - that, when incorporated into the mosaic penA 35 allele, confer essentially all of the increased resistance of penA41. Mapping these onto the crystal structure of PBP 2 shows that A311V and T316P are close to the active-site nucleophile, Ser310, that forms the acyl-enzyme complex, while Thr483 lies on a loop close to the active site and is predicted to interact with the carboxylate of the beta-lactam antibiotic. These three mutations have thus far only been described in penA41, but dissemination of these in other mosaic alleles would spell the end of ceftriaxone as an effective treatment for gonococcal infections.

\section{P1.020 PHENOTYPIC AND GENETIC CHARACTERIZATION OF THE FIRST THREE CASES OF EXTENDED-SPECTRUM CEPHALOSPORIN RESISTANT NEISSERIA GONORRHOEAE INFECTION IN SOUTH AFRICA AND ASSOCIATION WITH CEFIXIME TREATMENT FAILURE}

doi:10.1136/sextrans-2013-051184.0241

${ }^{1}$ L Y E Gumede, ${ }^{2} \mathrm{M}$ Unemo, ${ }^{3} \mathrm{C}$ Sriruttan, ${ }^{2} \mathrm{D}$ Golparian, ${ }^{1} \mathrm{E}$ E Müller, ${ }^{4} \mathrm{~K}$ Rebe, ${ }^{5} \mathrm{D}$ Fick, ${ }^{6} \mathrm{~J}$ de Wet, ${ }^{3} \mathrm{~J}$ Coetzee, ${ }^{17,8} \mathrm{D}$ A Lewis. ${ }^{1}$ National I nstitute for Communicable Diseases, Johannesburg, South Africa; ${ }^{2}$ WHO Collaborating Centre for Gonorrhoea and other STIS, Swedish Reference Laboratory for Pathogenic Neisseria, Örebro University Hospital, Örebro, Sweden, Örebro, Sweden; ${ }^{3}$ Department of Clinical Microbiology, Ampath National Laboratory Services, Centurion, South Africa, Centurion, South Africa; ${ }^{4}$ Anova Health Institute, Ivan Toms Centre for Men's Health, Cape Town, South Africa, Cape Town, South Africa; ${ }^{5}$ Meldene Medicross Clinic, Johannesburg, South Africa, Johannesburg, South Africa; ${ }^{6}$ Springs Medicross Clinic, Springs, South Africa, Springs, South Africa; ${ }^{7}$ Department of Internal Medicine, University of the Witwatersrand, Johannesburg, South Africa, Johannesburg, South Africa; ${ }^{8}$ Division of Medical Microbiology, University of Cape Town Medical School, Cape Town, South Africa, Cape Town, South Africa

Objectives To describe the phenotypic and genetic characteristics of the first three cases of extended-spectrum cephalosporin (ESC) resistant Neisseria gonorrhoeae in South Africa which were associated, in one case, with a verified cefixime treatment failure.

Methods Three ESC resistant $N$. gonorrhoeae isolates were cultured from the urethral discharge of three men-who-have-sexwith-men (MSM), two residing in Johannesburg and one in Cape 